\title{
Erratum to: Summer monsoon rainfall trends in the Indian Himalayan region
}

\author{
Sandipan Mukherjee • Rajesh Joshi • Ram C. Prasad • \\ Subhash C. R. Vishvakarma • Kireet Kumar
}

Published online: 25 November 2014

(C) Springer-Verlag Wien 2014

\section{Erratum to: Theor Appl Climatol \\ DOI 10.1007/s00704-014-1273-1}

The original version of this article unfortunately contained mistakes. The latitude and longitude values of Fig. 4a(i-vi) should be read as: $31.0-36.0 \mathrm{~N}$ and 73.0-80.0 E; for Fig. $4 \mathrm{c}(\mathrm{i}-$ vi) as: $23.0-29.5 \mathrm{~N}$ and $88.0-97.5 \mathrm{E}$. The italic values of Table 3 are for: Year 1991-2000: $\mathrm{CH}$ : Average rainfall trend

(0.18); Year 1951-1980: EH: Average rainfall trend (-0.04); Year 1951-1980: EH: Maximum rainfall trend (-0.43); Year 1951-1980: CH: Extreme rainfall trend (-0.20); Year 19511980: EH: Extreme rainfall trend (-0.29); Year 1951-2007: WH: Maximum rainfall trend (-0.12); and Year 1951-2007: WH: Extreme rainfall trend $(-0.08)$.

The corrected Fig. 4 and Table 3 are given below.

The online version of the original article can be found at http://dx.doi.org/ 10.1007/s00704-014-1273-1.

S. Mukherjee $(\bowtie) \cdot$ R. Joshi $\cdot$ R. C. Prasad · S. C. R. Vishvakarma

K. Kumar

G.B. Pant Institute of Himalayan Environment and Development,

Kosi-Katarmal, Almora 263 643, Uttarakhand, India

e-mail: sandipanmukherjee@hotmail.com 
Figure 4 Percentage departure of the decadal JJAS rainfall from the long-term average

(1951-2007) is represented for a WH, b CH, and $\mathbf{c} \mathrm{EH}$. The notations (i) to (vi) indicate six decadal periods as: 1951-1960, 1961-1970, 1971-1980, 1981-1990, 1991-2000, and 2001-2007, respectively
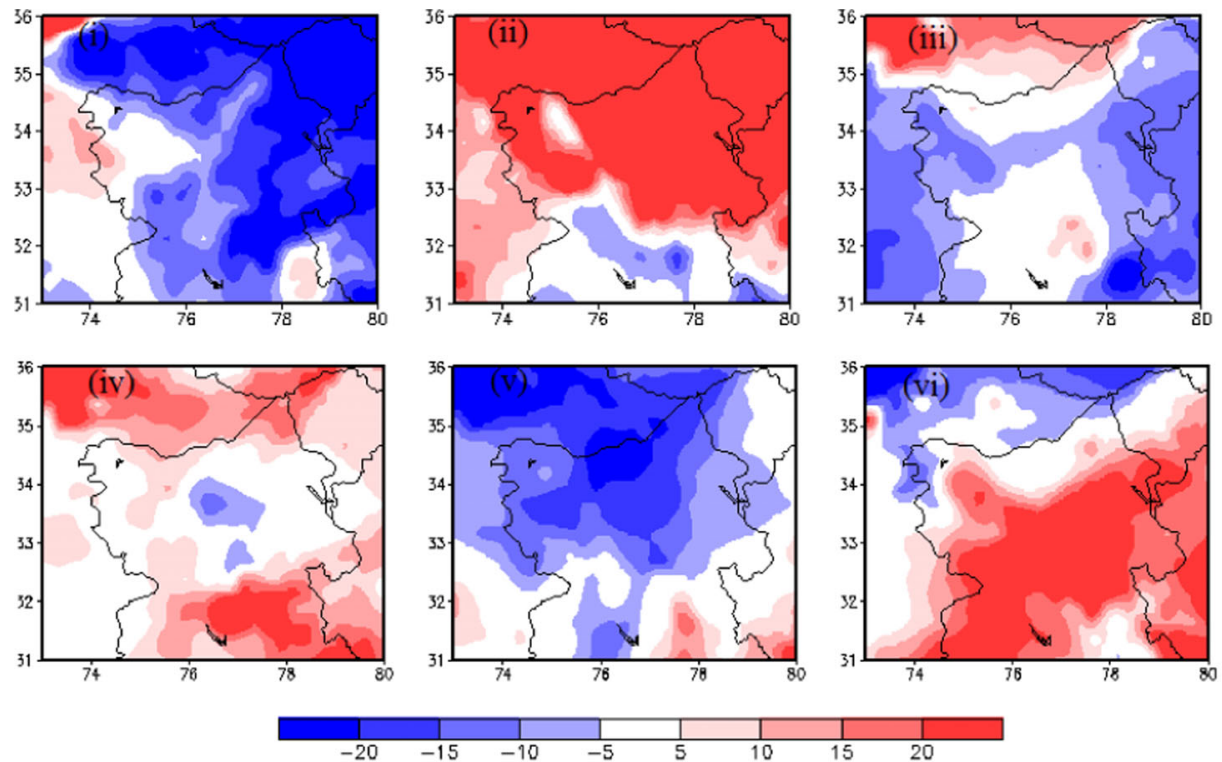

(a) $\mathrm{WH}$
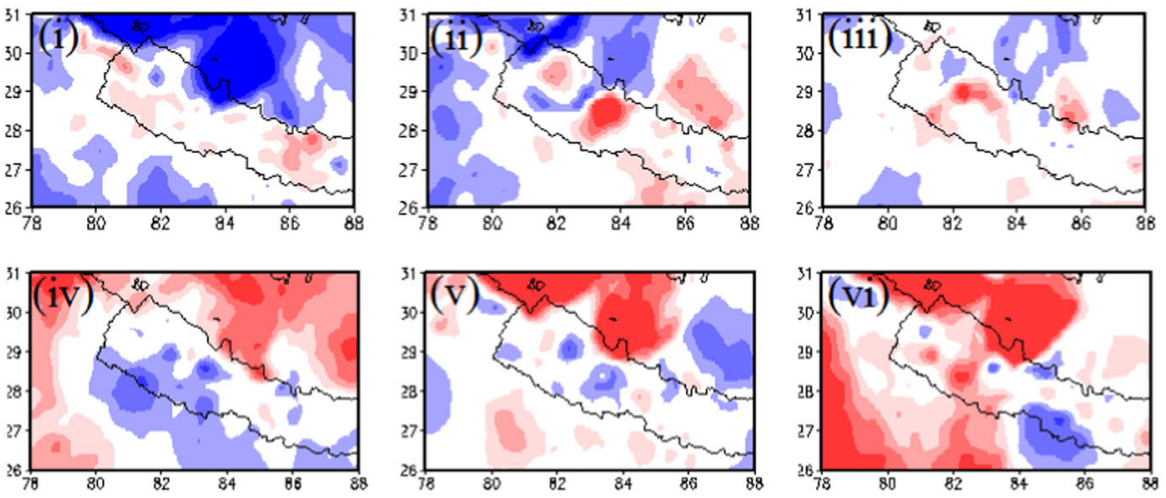

(b) $\mathrm{CH}$
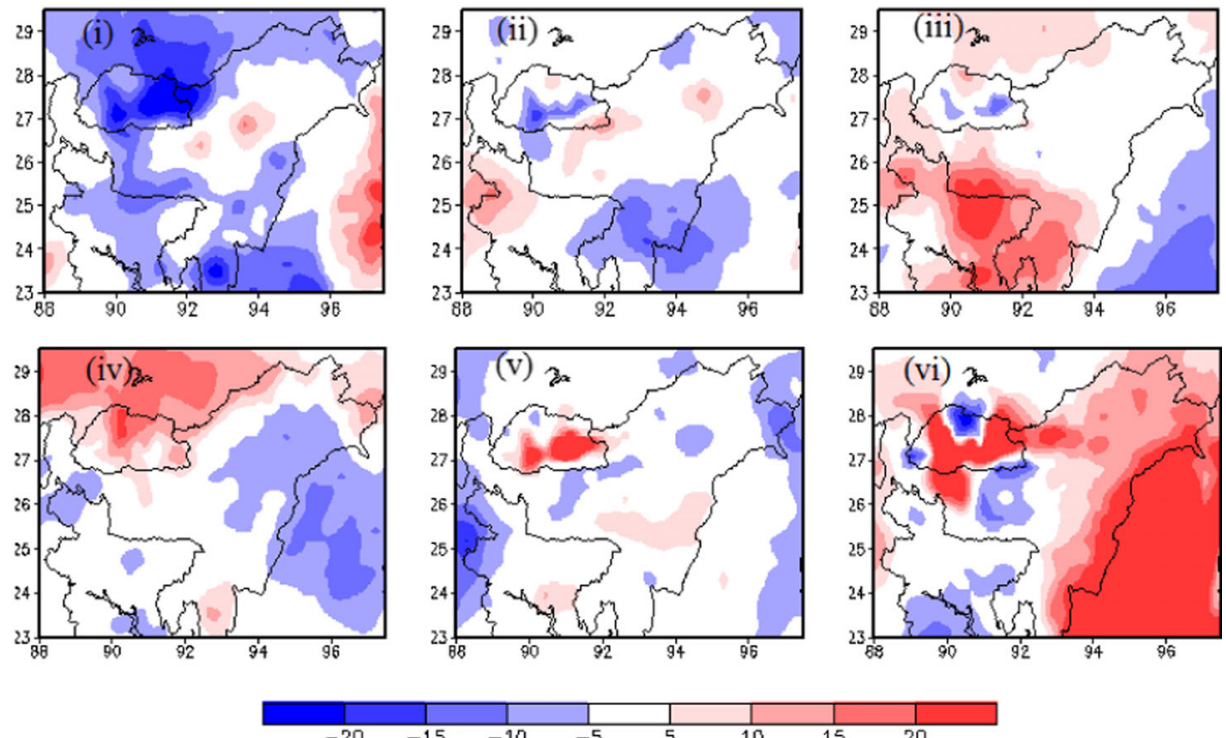

(c) $\mathrm{EH}$ 
Table 3 Sen's estimator of slope $(\mathrm{mm} /$ day) for the seasonal average, maximum, and extreme rainfall events

Values in italics are statistically significant at a $p$ value $<=0.05$

\begin{tabular}{|c|c|c|c|c|c|c|c|c|c|}
\hline \multirow[t]{3}{*}{ Period } & \multicolumn{3}{|c|}{ Average rainfall trend } & \multicolumn{3}{|c|}{ Maximum rainfall trend } & \multicolumn{3}{|c|}{ Extreme rainfall trend } \\
\hline & \multicolumn{3}{|c|}{ Region } & \multicolumn{3}{|c|}{ Region } & \multicolumn{3}{|c|}{ Region } \\
\hline & $\mathrm{NH}$ & $\mathrm{CH}$ & $\mathrm{EH}$ & $\mathrm{NH}$ & $\mathrm{CH}$ & $\mathrm{EH}$ & $\mathrm{NH}$ & $\mathrm{CH}$ & $\mathrm{EH}$ \\
\hline $1951-1960$ & 0.10 & 0.03 & -0.09 & 1.32 & 0.25 & -1.31 & 0.33 & 0.00 & 0.00 \\
\hline $1961-1970$ & -0.04 & -0.06 & 0.10 & -0.49 & -0.28 & 2.11 & -0.14 & -0.20 & 0.88 \\
\hline $1971-1980$ & 0.01 & 0.00 & -0.02 & -0.36 & 0.32 & 0.24 & -0.14 & -0.33 & 0.44 \\
\hline $1981-1990$ & 0.04 & 0.01 & 0.08 & 0.62 & -0.29 & -0.84 & 0.38 & -0.80 & -0.83 \\
\hline $1991-2000$ & 0.02 & 0.18 & 0.06 & 0.22 & 1.07 & 1.89 & 0.00 & 1.00 & 0.75 \\
\hline $2001-2007$ & 0.02 & -0.10 & -0.22 & 0.60 & -0.12 & -0.25 & 0.50 & -0.50 & 0.80 \\
\hline $1951-1980$ & 0.00 & -0.01 & -0.04 & -0.05 & -0.13 & -0.43 & -0.08 & -0.20 & -0.29 \\
\hline $1980-2007$ & 0.00 & -0.01 & -0.05 & -0.08 & -0.02 & -0.06 & 0.00 & 0.00 & 0.10 \\
\hline $1951-2007$ & 0.00 & 0.00 & -0.01 & -0.12 & 0.00 & -0.01 & -0.08 & -0.04 & 0.00 \\
\hline
\end{tabular}

